\title{
The Effects of Graphical Fidelity on Player Experience
}

\author{
Kathrin M. Gerling, Max Birk, Regan L. Mandryk, Andre Doucette \\ Interaction Lab, University of Saskatchewan \\ 110 Science Place \\ Saskatoon SK S7N 5C9, Canada \\ +13069662327 \\ \{firstname.lastname\}@usask.ca
}

\begin{abstract}
Graphical assets in video games have become increasingly complex over the years, but little is known about their effect on player experience (PX). In this paper, we present results of a controlled study with 48 participants comparing how abstract and stylized graphics influence player experience in casual games. Our results show that high-fidelity graphics result in a more positive impression of the game. However, we also show that many effects are only present in the game with a more challenging mechanic. This shows that casual games can be compelling and enjoyable to play despite simplistic graphics, suggesting that small game developers and researchers need not focus on elaborate visuals to engage players.
\end{abstract}

\section{Categories and Subject Descriptors \\ K.8.0 [Personal Computing]: General - Games.}

\section{General Terms}

Measurement, Design, Experimentation.

\section{Keywords \\ Player Experience, Casual Games, Game Graphics.}

\section{INTRODUCTION}

Over the last few decades of game design and development, the fidelity of graphical assets in computer and video games has changed from basic pixel art to complex 3D models, complete with textures and lighting. Game mechanics have also become more complex, creating an environment where some of the original computer games are viewed as casual today. Researchers have focused on how game mechanics affect the player (e.g., [3]); however, there has been little investigation into how the fidelity of game graphics affects player experience (PX) - especially under conditions where game mechanics are kept consistent. This is an important question to consider for a number of reasons.

First, there is a tradition in game development of testing mechanics in an iterative process, with increasing fidelity of the prototype through the development lifecycle [10]. It is important to understand whether (and in what kinds of ways) the fidelity of graphics used in the lower-fidelity prototypes affect player

Permission to make digital or hard copies of all or part of this work for personal or classroom use is granted without fee provided that copies are not made or distributed for profit or commercial advantage and that copies bear this notice and the full citation on the first page. To copy otherwise, or republish, to post on servers or to redistribute to lists, requires prior specific permission and/or a fee.

MindTrek 2013, October 1-4'2013, Tampere, FINLAND.

Copyright 2013 ACM 978-1-4503-1993-8/13/10 ...\$10.00. response to the mechanic under evaluation. For example, if we can determine that fidelity of graphics affect player immersion, but not player motivation, then games user researchers have license to test new mechanics early with very basic graphical prototypes. Second, for those who use games as research tools, but do not have the budgets to create slick research prototypes, understanding how players respond to lower-fidelity graphics in a game can help inform where scarce resources should be committed in creating game-based research prototypes. And third, there is a common perspective in game design that games can be described as the inter-relation of layers; the MDA framework defines mechanics (e.g. rules, algorithmic action), dynamics (e.g. interaction of mechanics), and aesthetics (e.g. the visual representation of mechanics and dynamics, and the resulting emotion in the player) as the core components of games [14]. Understanding the relationship between the layers of this framework, and how changes in one layer affect another is important for advancing the theory of game interaction.

To investigate how the fidelity of graphics affects player experience, we developed two casual games with different mechanics and dynamics, and compared player experience in versions with both low-fidelity graphics (abstract) and highfidelity graphics (stylized) in a user study with 48 participants. Findings suggest that high-fidelity graphics result in an overall increase in the sense of immersion, resulting in a more positive impression of the game. However, we also show that differences due to fidelity in the intrinsic motivation to play, the feeling of control over the game, and the resulting feeling of competence in the player are only present in the game with a more challenging mechanic. That is, a less demanding mechanic does not need highfidelity graphics to maintain a quality player experience.

This result has several implications for game design and development. First, it suggests that casual games can be compelling and enjoyable to play, even if few development resources are devoted to complex graphics - which is good news for indie and end-user developers. Second, it suggests that in the production cycle of casual games, the evaluation of core mechanics in low-fidelity prototypes is reliable, smoothing the iterative game development process for casual game studios, especially those with small development teams and budgets. And finally, it suggests that researchers who use simple game mechanics to study games or game-based interaction can reliably deploy research prototypes without a need to commit significant resources to graphical assets.

\section{RELATED WORK}

Much work investigating the effects of graphical quality on player experience has been carried out in the field of media psychology with a focus on media effects. Although this line of work does not investigate player experience from the perspective of game design, it holds implications for the study presented in this paper. 


\subsection{Graphics in Media Effects Research}

Research in psychology has focused on the impact of graphical quality on media recipients from the perspective of media effects research, investigating how visuals affect our emotional experience. Two main aspects in this area are the effects of image quality on internal states, e.g., presence, the sense of being part of an environment, being involved, and perceiving something as real [4], and on behaviour.

First efforts in this field addressed effects of television image quality on the viewer's experience. Detenber et al. [8] investigated whether colour has an impact of the emotional experience of viewers, and found that participants preferred coloured clips over monochrome versions; however, they could not find differences in physiological measures. In another study comparing HDTV and analog television images, Bracken and Botta [4] found that participants viewing HDTV content reported a higher degree of presence than participants viewing analog images.

Based on these results, similar studies were conducted to investigate the effects of the graphical quality of video games on players. Ivory and Kalyanaraman [15] examined how differences in graphical quality of the arcade-style games Zombie Raid (low graphical quality) and The House of the Dead 2 (high graphical quality), as well as Diamonds 3D (low graphical quality) and 3D Arkout (high graphical quality) affect aggression. Their results show that participants in high graphical quality conditions perceived a higher level of presence, involvement and arousal; however, the study reports no significant impact of graphical quality on aggression measures. Along these lines, Krcmar et al. [16] investigated the effects of graphical realism on attention, retention and aggression. As stimulus, they applied the firstperson shooting (FPS) games Doom 1 (low graphical realism) and Doom 3 (high graphical realism). Their results show that participants perceived Doom 3 as more realistic than Doom 1, reported a higher level of presence when playing Doom 3, and showed higher aggression scores. Following up on these results, Bracken and Skalski [6] examine the effects of image quality on presence and video games. In their study, they apply the FPS game Perfect Dark Zero in an HDTV and NTSC version; while the results show no significant differences in presence, the authors report significant differences on one of its subscales, immersion, suggesting that higher image quality leads to higher immersion, a relevant construct in games user research.

On a general level, the results of these studies show that image quality has an impact on the experience of media recipients, and that this hypothesis is also true when investigating the effects of image quality in video games. However, because these studies have been conducted in the realm of media effects research, there are certain limitations that need to be addressed for results to be applicable to inform the work of game designers wishing to create immersive interactive experiences. First, existing work does not investigate impact of graphical quality of games in a tightly controlled manner. By choosing to apply commercially available games rather than designing specific game-based research tools, potential confounds that may affect player experience are introduced (e.g., differences in control schemes or slightly different game mechanics), or the change in visual quality may not be substantial enough to induce differences in player experience (e.g., when providing analog and HDTV image quality). For example, the study by Krcmar et al. [16] does not address the potential impact of different control schemes on perceived realism; while Doom 3 supports mouselook, Doom 1 only allows players to apply the mouse to adjust the horizontal crosshair position, limiting the player's ability of freely navigating their avatar, an aspect which may have influenced player experience. Second, existing studies focus on outlining basic effects of image quality on presence and aggression. However, results from games user research suggest that player experience is a complex phenomenon. To provide further insights into the impact of image quality on player experience, further research accounting for other fundamental aspects of video games, e.g., game mechanics and control schemes, are necessary. Therefore, we suggest to investigate the effects of graphical quality on player experience from the perspective of games user research to highlight which aspects of player experience are affected by graphical quality, and to provide practical recommendations that can inform the work of game designers.

\subsection{Graphics and Games User Research}

Games user research has applied concepts from psychology to decompose player experience with the goal of helping game developers make informed design decisions. Ermi and Mäyrä [9] examine immersion in video games and suggest that adaptions are necessary for the concept to be applicable to video games. Based on Csikszentmihalyi's concept of flow, Sweetser and Wyeth [24] develop the GameFlow model, an approach which leverages the idea of creating an optimal experience by matching skill and challenge to apply it to the design of video games. Building on previous work investigating flow and immersion in video games and focus group interviews, Poels et al. [18] propose a model of player experience consisting of the nine dimensions enjoyment, flow, imaginative immersion, sensory immersion, suspense, competence, negative affect, control, and social presence, particularly highlighting the variety of aspects that contribute to the overall experience when engaging with games. Ryan et al. [21] propose a model building on psychological constructs. They apply Self Determination Theory to analyze player experience and identify intrinsic motivation, competence, autonomy, relatedness, presence, and intuitive controls as relevant components.

These examples show that player experience is a complex phenomenon, and existing work in games user research has outlined factors that contribute to the overall experience. In that respect, little work has been carried out in games user research. A first approach by Cheng and Cairns [7] investigates the impact of changes in game mechanics and graphical realism on immersion. Using a modification of Unreal Tournament 2003, they changed avatar graphics, game graphics, and game physics. Results show that participants noticed changes in graphical realism, but not in game physics, and that participants reported a consistent game experience across all conditions. Therefore, when investigating the impact of graphical quality on player experience, research beyond mere psychological effects of video games on players is necessary to account for interaction effects between different game elements (e.g., mechanics, controls, and graphics) that may influence player experience. Andersen et al. [1] present a largescale study that examines how aesthetics - music, sound, and animations - influence player behaviour in casual games. They conclude that the inclusion of animations has a positive effect on players: their results show that participants in the animation condition advanced further in the game and played significantly longer than participants in the condition that did not include animations. However, the authors note that animations did not only have an aesthetic value, but also provided player feedback. They do not provide insights into whether participants in the 
condition without animations received other feedback on the state of the game world; however, they hypothesize that players without animations experienced lower levels of usability. Finally, a study investigating the impact of graphical fidelity in motion-based games for older adults suggests that there are differences in player experience between abstract and detailed representations, but that the level of detail does not influence PX [23].

Building on these results and an exploratory study investigating the impact of graphical representation on player experience in Rockband 2 (section 3.1), the work presented in this paper presents a controlled study that investigates the impact of graphical quality on PX. We investigate whether PX is affected by graphical fidelity of games in two casual gaming scenarios; while we do make adaptions to essential parts of the game, the amount of feedback players receive across conditions is consistent, and game mechanics do not change within the low- and high-fidelity versions of each game. Additionally, by including games with different core mechanics, we further examine the relationship between visuals, game mechanics, and PX.

\section{STUDY: GRAPHICAL FIDELITY AND PLAYER EXPERIENCE}

In this section, we describe our approach for examining the impact of graphical fidelity on player experience.

\subsection{Background: The Rockband Chronicles}

Many games have elaborate graphics, and while some of them are essential to the game (e.g., game objects that the player can interact with), others are a way of adding to PX by reinforcing the idea of the game. For example, in the Rockband series, the only graphical components required to play are the frets in the center of the screen (Figure 1, left). Environmental graphics (cheering crowds, avatars of band members, pyro techniques) are all extraneous graphics that are not required to complete the game.

Based on the concept of chart junk [2], graphical elements that decrease the data-to-ink ratio in information visualizations, we hypothesized that game junk may contribute to a player's experience of the game, potentially making games more fun, more immersive, and increase a player's suspension of disbelief. To explore how this game junk affects player experience, we conducted an exploratory study. We created a physical cardboard template to cover all but the guitar frets, and laid it over the monitor (Figure 1, right), creating two play conditions - with the template (no junk) and without the template (with junk). Sixteen participants played Rockband 2 in the junk and no junk conditions (counterbalancing order of presentation). Participants played the game on a Tobii $60 \mathrm{XL}$ eye-tracker, which gathered their gaze fixations. They completed post-condition and post-experiment questionnaires to gather their subjective experience. We also gathered physiological data for the first eight participants to analyze player experience. Surprisingly, players did not report differences in fun, frustration, or perceived success of the two conditions, even though there was a physical piece of cardboard blocking their view in the no junk condition. Physiological data did not show any difference in overall tonic experience or phasic responses to in-game events. This lack of differences was not dependent on the expertise of the player (expert or novice), or whether they were in a social environment (alone or playing with a friend watching). The fact that we saw no differences may be explained by the eye-tracking results, which showed that players looked at game junk an average of 1.5 times per song.
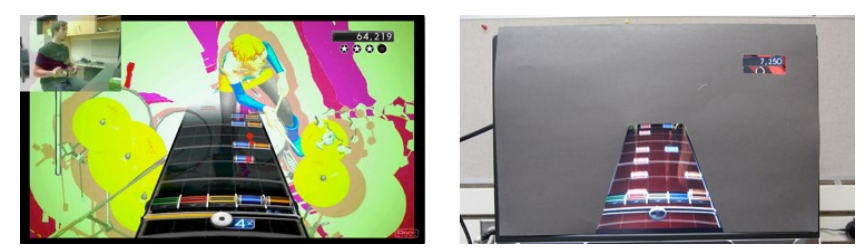

Figure 1. Rockband study, condition A with full effects (left) and condition $B$ with reduced visuals (right).

Although it was surprising that the absence of game junk did not affect player experience as measured subjectively or objectively, there are a few reasons why this may be the case. First, the game mechanic of Rockband (the production of music) is very compelling, so the game is still fun even in the absence of the game junk. Second, the controller used for Rockband provides additional value to player experience by aiding in the willing suspension of disbelief. Third, players focus on the graphics required for gameplay, and rarely fixate on game junk; if players do not attend to the junk, then the junk is unlikely to affect their experience. To isolate the impact of graphical fidelity in a controlled experiment, we created two games that integrate graphics into mechanics, forcing players to attend to their fidelity.

\subsection{Experiments using Integrated Graphics}

To evaluate how graphical fidelity influences player experience when players are forced to attend to the graphics, we developed two custom games, Sparkly Frogger and Candy Kids. The visual styles of our games build on a framework for visual styles for serious games by McLaughlin et al. [17], who suggest a distinction between simplified, stylized, and realistic visual styles. For each game, we created a high-fidelity version, including detailed sprites that allow players to make a connection with the game premise. We also created low-fidelity versions of both games in which sprites were replaced with solid shapes. These low-fidelity sprites retained the bounding area of the high-fidelity sprites (and so did not affect gameplay), but were shaded in the dominant solid colour of the high-fidelity sprite (Figure 2). We called these stylized graphics and abstract graphics, respectively.

\subsubsection{Sparkly Frogger}

Sparkly Frogger is a clone of the popular arcade game Frogger. In the game, players are represented by a frog; starting out at the bottom of the screen, they have to cross two lanes of moving objects in order to reach one of five pods at the top of the screen. Once all five pods have been reached, the player advances to the next game level; game difficulty is increased in each successive level through faster moving cars, and sinking lily pads and logs. In the high-fidelity version (Figure 2, left), the avatar is clearly identifiable as a frog, and other game elements (e.g., cars, pods, logs, lily pads, and lanes) can be recognized by players. In the low-fidelity version (right), graphical detail is greatly reduced, and connections between game elements and the premise of the game are less obvious. Sparkly Frogger is played with an Xbox 360 gamepad; the game uses the D-pad to let players move the frog up, down, to the right, or to the left. The frog dies if it hit by a car (or its low-fidelity counterpart), or misses the lily pad or log (or their low-fidelity counterparts). Players get three lives before losing the game, resulting in a restart.

\subsubsection{Candy Kids}

Candy Kids is a casual game in which players are challenged to collect candy and feed it to a child. In the game, candy moves across the upper area of the screen and can be picked up by 

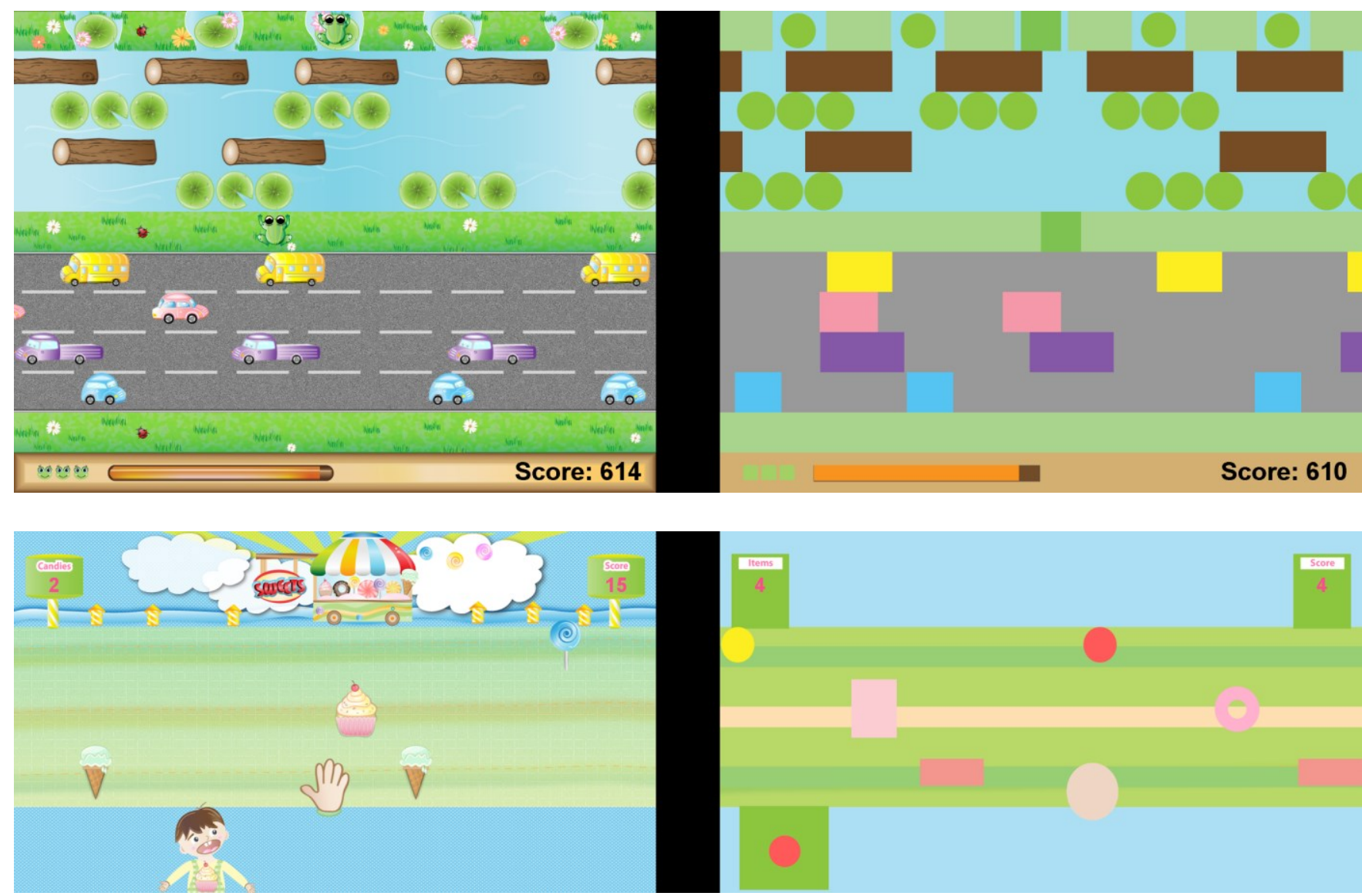

Figure 2. High-fidelity stylized graphics (left) and low-fidelity abstract graphics (right) in the versions of Sparkly Frogger (top) and Candy Kids (bottom).

moving the player cursor (represented by a virtual hand) overtop of the candy. After picking up candy, players can feed it to the child by moving their avatar towards the child. Challenge is created by switching the type of candy the child prefers after each round of feeding; an icon of the currently accepted candy is displayed on the child's clothing, and players have to match it to increase their score and avoid a penalty. Candy Kids is played using the Xbox 360 controller. Similar to Sparkly Frogger, we created high-fidelity and low-fidelity versions of Candy Kids. In the high-fidelity version of the game, the game elements are wellstyled and reinforce the premise. In contrast, the graphics in the low-fidelity version contain no references to candy or children, and are abstract representations of their high-fidelity counterparts.

\subsection{Participants and Procedure}

The study was conducted with 48 participants from the local University ( $49 \%$ female, mean age $=24.2, \mathrm{SD}=3.23)$. Students were recruited via mailing lists and notices on bulletin boards. Most participants played games on a regular basis (86\%) and were familiar with an Xbox 360 Gamepad (61\%). Participants began by completing an informed consent and a questionnaire about their current affective state. Participants played either Sparkly Frogger or Candy Kids using the Xbox 360 Gamepad, but played the game with both levels of Fidelity [stylized, abstract] presented in counter-balanced order (mixed model). After finishing a condition, participants completed questionnaires assessing their experience. Finally, participants completed a demographic questionnaire.

\subsection{Measures}

To understand how fidelity of a casual game affects play, we analyzed player response to validated scales, and player behaviour via game metrics. We focused on player experience, decomposing experience into underlying constructs, effort required to play, game difficulty, and performance.

Player Experience. Intrinsic Motivation was assessed using the 18-item Intrinsic Motivation Inventory [19], which has been used to evaluate experience with video games (e.g., [21]). A series of items are rated on 5-point Likert-scale, ranging from 1 (not at all) to 5 (quite a bit). We report on the interest-enjoyment subscale of the inventory, which reflects intrinsic motivation. Positive Affect and Negative Affect were assessed using the Positive Affect Negative Affect Schedule-Expanded (PANAS-X) [26]. In the PANAS-X, participants are asked to agree with 20 emotion adjectives on a Likert-scale ranging from 1 (very slightly or not at all) to 5 (extremely). The PANAS-X has been used to evaluate the enjoyment of video games (e.g., [19]).

Need Satisfaction. The Player Experience of Need Satisfaction Scale (PENS) [21] investigates game experience from the perspective of Self-Determination Theory [20], and has been used successfully to evaluate games (e.g., [21]). We used PENS after each condition to assess if the game satisfied the players' needs for Competence, Autonomy, Relatedness, Immersion and Intuitive Controls. A series of statements was agreed with using a 5-point Likert-scale from 1 (not at all) to 5 (quite a bit). 
Workload and Performance. NASA Task Load Index (TLX) [25] was designed to assess workload during tasks. The TLX includes the six subscales: Mental Demands, Physical Demands, Temporal Demands, Own Performance, and Frustration. We report the combined workload score as described by [13]. Performance metrics were calculated using data from game logs. The log files included key input (left, right, up, down), and the number of deaths for Sparkly Frogger. The error metric was defined as the ratio of deaths over key input. For Candy Kids, grabbed and fed items were logged with respect to the currently accepted candy. The error metric was defined as the ratio of the sum of mismatched candy grabbed over the sum of all candy grabbed.

\subsection{Results}

We first present results for player experience measured by scales for visual rating, intrinsic motivation, and positive affect. We then explore how fidelity affects need satisfaction. Finally, we investigate the differences of difficulty using measures for workload and performance. Measures were analyzed using RMANOVAs with fidelity (high, low) as a within-subject factor and game (Sparkly Frogger, Candy Kids) as a between-subjects factor. All statistical tests were performed using SPSS 20. Post-hoc tests with Bonferroni correction were performed for multiple comparisons and tested for significance at $\alpha=0.05$. Descriptive statistics (means and standard deviations) and the results of statistical tests $\left(F, p\right.$, and $\left.\eta^{2}\right)$ are shown in Table 1.

\subsubsection{Player Experience}

To determine whether our experimental manipulation of fidelity was interpreted by players, we compared participant ratings of visual appearance (collected on a 5-point scale, with $1=$ low and $5=$ high). The results show a significant main effect for fidelity (abstract perceived as less visually appealing than stylized), but no main effect of game or interaction of game and fidelity. These results support that the fidelity differences were perceived as expected in both games. The results for intrinsic motivation show a significant main effect for fidelity (higher intrinsic motivation in stylized), but not for game. However, the significant interaction, clarifies that the higher intrinsic motivation in the stylized condition is significant in Frogger, but not in Candy Kids. The results for positive affect reveal a significant main effect for fidelity (stylized more positive than abstract) and game (Candy Kids more positive than Sparkly Frogger). There was no significant interaction between game and fidelity.

\subsubsection{Need Satisfaction}

In a second step, we analyzed the need satisfaction that both games provided for players to further investigate the effects of fidelity and type of game.
Competence. There were significant main effects of fidelity (more perceived competence in stylized) and game (more perceived competence in Candy Kids); however, the significant interaction between game and fidelity shows that the higher perceived competence in the stylized condition is significant for Sparkly Frogger, but not for Candy Kids.

Autonomy. There was a significant main effect of fidelity (more perceived autonomy in stylized), but only a marginal effect for game (more perceived autonomy in Candy Kids). However, a significant interaction between game and fidelity shows that the higher perceived autonomy in the stylized condition is significant for Sparkly Frogger, but not for Candy Kids.

Relatedness. There was a significant main effect of fidelity (more perceived relatedness in stylized), but no effect of game, or interaction between game and fidelity.

Immersion. There was a significant main effect of fidelity (more immersion in stylized), but no effect of game, or interaction between game and fidelity.

Intuitive Controls. There was no significant main effect of fidelity or game; an interaction between fidelity and game shows that the ratings of intuitive control were higher in the stylized condition for Sparkly Frogger, but not for Candy Kids.

\subsubsection{Workload and Performance}

When considering the differences in the enjoyment and need satisfaction in the experiment, it is important to consider whether the results can be attributed in part to variations in difficulty or performance. The overall workload measured through the NASATLX shows no effect for fidelity, but does show a significant main effect for game, with higher workload for Sparkly Frogger. There was no interaction of fidelity with game.

The error metric (as defined in section 3.4) shows no effect for fidelity, but does show a significant main effect for game, with higher percentage of errors in Candy Kids. There was no interaction of fidelity with game. The error metric results for game need to be interpreted with caution though, as they were defined similarly for two very different mechanics - there were more errors in candy grabs as a percentage of all grabs than there were frog deaths as a percentage of all moves. This will yield differences simply because of the spread in the overall number of frog moves and candy grabs. If we look at the absolute number of errors made in game, Sparkly Frogger resulted in an average of 18.79 frog deaths, whereas Candy Kids resulted in an average of 2.08 incorrect candy selections. The point of interest for our analysis is that there is no effect of fidelity and no interaction of fidelity with game, showing that participants did not commit more

Table 1. Means, Standard Deviation, Effects for used scales by game (Frogger, Candy Kids) and fidelity (high, low).

\begin{tabular}{|c|c|c|c|c|c|c|c|}
\hline & \multicolumn{2}{|c|}{ Frogger } & \multicolumn{2}{|c|}{ Candy Kids } & \multirow[b]{2}{*}{ Effect of Game } & \multirow[b]{2}{*}{ Effect of Fidelity } & \multirow[b]{2}{*}{ Game x Fidelity } \\
\hline & High & Low & High & Low & & & \\
\hline Pos. Affect & $2.77(0.81)$ & $2.38(0.91)$ & $3.12(0.8)$ & $3.02(0.65)$ & $\mathrm{F} 1,46=5.49, \mathrm{p}<.05, \eta 2=.11$ & $\mathrm{~F} 1,46=6.85, \mathrm{p}<.05, \eta 2=.13$ & $\mathrm{~F} 1,46=2.38, \mathrm{p}=.13$ \\
\hline Neg. Affect & $1.47(0.6)$ & $1.5(0.63)$ & $1.3(0.41)$ & $1.35(0.52)$ & $\mathrm{F} 1,46=1.27, \mathrm{p}=.27$ & $\mathrm{~F} 1,46=.42, \mathrm{p}=.52$ & $\mathrm{~F} 1,46=.01, \mathrm{p}=.92$ \\
\hline Int. Mot. & $3.63(0.83)$ & $2.51(0.99)$ & $3.33(0.83)$ & $3.25(0.75)$ & $\mathrm{F} 1,46=2.79, \mathrm{p}=.28$ & $\mathrm{~F} 1,46=16.27, \mathrm{p}<.001, \eta 2=.26$ & $\mathrm{~F} 1,46=12.49, \mathrm{p}<.001, \eta 2=.21$ \\
\hline Competence & $3.42(0.86)$ & $2.71(1.26)$ & $4(0.64)$ & $3.96(0.68)$ & $\mathrm{F} 1,46=16.02, \mathrm{p}<.001, \eta 2=.26$ & $\mathrm{~F} 1,46=9.70, \mathrm{p}<.01, \eta 2=.17$ & $\mathrm{~F} 1,46=7.63, \mathrm{p}<.01, \eta 2=.14$ \\
\hline Autonomy & $2.58(0.91)$ & $1.83(0.77)$ & $2.61(1.06)$ & $2.6(0.69)$ & $\mathrm{F} 1,46=3.94, \mathrm{p}=.053, \eta 2=.08$ & $\mathrm{~F} 1,46=6.28, \mathrm{p}<.01, \eta 2=.12$ & $\mathrm{~F} 1,46=5.83, \mathrm{p}<.05, \eta 2=.11$ \\
\hline Relatedness & $2.32(1.05)$ & $1.75(0.63)$ & $2.17(0.98)$ & $2.03(0.81)$ & $\mathrm{F} 1,46=0.91, \mathrm{p}=.76$ & $\mathrm{~F} 1,46=5.68, \mathrm{p}<.05, \eta 2=.11$ & $\mathrm{~F} 1,46=2.10, \mathrm{p}=.15$ \\
\hline Immersion & $2.5(0.96)$ & $1.74(0.51)$ & $2.43(0.94)$ & $2.11(0.61)$ & $\mathrm{F} 1,46=0.62, \mathrm{p}=.43$ & $\mathrm{~F} 1.46=18.38, \mathrm{p}<.001, \eta 2=.29$ & $\mathrm{~F} 1,46=3.15, \mathrm{p}=.08$ \\
\hline Int.-Con. & $4.17(0.56)$ & $3.74(1.24)$ & $4.29(0.65)$ & $4.38(0.67)$ & $\mathrm{F} 1,46=3.27, \mathrm{p}=.08$ & $\mathrm{~F} 1,46=2.54, \mathrm{p}<.01$ & $\mathrm{~F} 1,46=5.57, \mathrm{p}<.02, \eta 2=.11$ \\
\hline Visual & $7.38(2.04)$ & $3.58(2.06)$ & $6.67(2.41)$ & $3.83(2.16)$ & $\mathrm{F} 1,46=.184, \mathrm{p}=.67$ & $\mathrm{~F} 1,46=102.10, \mathrm{p}<.001, \eta 2=.69$ & $\mathrm{~F} 1,46=2.14, \mathrm{p}=.151$ \\
\hline Workload & $8.63(3.34)$ & $9.71(3.36)$ & $5.53(3.22)$ & $5.56(3.51)$ & $\mathrm{F} 1,44=15.28, \mathrm{p}<.001, \eta 2=.26$ & $\mathrm{~F} 1,45=1.6, \mathrm{p}<.21$ & $\mathrm{~F} 1,45=2.6, \mathrm{p}<.11$ \\
\hline Error ratio & $.03(.02)$ & $.05(.04)$ & $.11(.06)$ & $.10(.10)$ & $\mathrm{F} 1,45=16.44, \mathrm{p}=.001, \eta 2=.26$ & $\mathrm{~F} 1,45=.07, \mathrm{p}=.79$ & $\mathrm{~F} 1,45=1.23, \mathrm{p}=.27$ \\
\hline
\end{tabular}


errors as a result of playing with stylized or abstract graphics. These results suggest that the aforementioned differences of experience as a result of our fidelity manipulation cannot be attributed to differences in workload or performance.

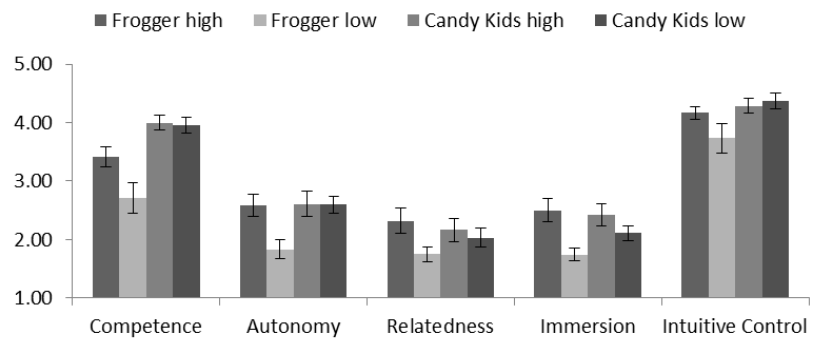

Figure 3: Means and Standard Deviation for PENS subscales for used games (Frogger, Candy Kids) by fidelity (high, low).

\subsection{Summary of Findings}

Our results show that fidelity of graphics does play a role in player experience, specifically in terms of positive affect, intrinsic motivation, competence, autonomy, relatedness, and immersion. This gives us a clear indication that fidelity is not only reflected in how a player experiences the visual appeal of a game, but also in how a player enjoys the game experience. Interestingly a number of metrics revealed an interaction between fidelity and game - for intrinsic motivation, competence, autonomy, and intuitive controls, there were effects of fidelity in Sparkly Frogger, but not in Candy Kids. This is of interest because it shows that fidelity matters, but not for all types of games.

In considering the differences between the two games, we can look to the variables that showed effects for the factor of game. Positive affect, competence, and autonomy were all experienced more during Candy Kids than Frogger. In addition, workload was rated as higher for Frogger and there were more frog deaths than incorrect candy selections, showing the Frogger was the more difficult game. However, the lack of differences in intuitive control between games suggest the input scheme was equally usable across conditions, and that usability issues did not interfere with player experience. Thus, differences in game difficulty were solely caused by game mechanics. So, we can say that Candy Kids was a less challenging game, which resulted in better performance, reflected in higher perceived competence and autonomy, and higher positive affect. It is also of importance to note the abstract graphics result in decreased player experience in Sparkly Frogger - it is not that the stylized graphics are producing a better experience (as compared to both fidelity conditions for Candy Kids), but that the abstract graphics are producing a worse experience (see Figure 3).

In summary, higher fidelity leads to more positive affect, higher relatedness, and increased immersion, regardless of game type. This suggests that adding objects to the games that players can relate to may improve experience by adding meaning to the game, making it more immersive and creating more positive sensations. On the other hand, a player's intrinsic motivation to play, along with their feelings of volition or control (autonomy) and competence, are only affected by the fidelity of graphics in the more challenging game that requires effort. In addition, the intuitiveness of controls was also only affected by fidelity in the more challenging game. These findings have two implications. First, they show that the graphical fidelity does influence player experience when graphics are integrated with game mechanics and players attend to them during gameplay. Second, our study shows that the way graphical fidelity affects player experience is influenced by difficulty, suggesting that easier games can provide a positive experience despite low-fidelity graphics, and that games including challenging mechanics may need to offer high-fidelity graphics to achieve the same level of player experience.

\section{DISCUSSION}

This paper investigates the effects of graphical fidelity in games on player experience. In this section, we discuss implications of our findings in the context of different elements of games, we outline how our results are relevant in casual game design, and we generalize our findings to be actionable for the broader game development community.

\subsection{Graphical Fidelity and Player Experience}

Our study provides the first controlled comparison of high- and low-fidelity game graphics, using two different game mechanics, and deploying questionnaires designed to provide detailed insights into aspects of player experience. This approach allows us to examine different ways in which graphics affect player experience, and to decompose player experience in a systematic and theoretically driven way.

Past work has looked at PX in games using broad perspectives (e.g., inquiring whether a game was 'fun') or in depth along a single dimension (e.g., examining the effects of visuals on immersion). Our results suggest that the effect of visual fidelity on player experience is multi-dimensional, and needs to be studied from different perspectives. Looking at the overall enjoyment resulting from the levels of fidelity, we find changes in affect, simply indicating that fidelity in graphics yields variations in fun and enjoyment. In contrast, intrinsic motivation explains whether the experienced fun is derived from personal interest, and in our study is not affected in the game with simple mechanics. This factor could also explain why we did not find results for fidelity in the Rockband studies - where graphics were not integrated into mechanics, but were extra (we called this game junk). If game objects yield no important information for the player, their graphical quality does not affect the experience. This is an interesting finding, because it gives insights in how players distinguish visual information in a game.

Looking at the factors that drive intrinsic motivation, we were interested in the motivational pattern that shows that competence and autonomy are influenced by the fidelity of graphics only in the more challenging game. Introducing a non-trivial conflict is a core mechanism in many games, but our results indicate that experienced competence and autonomy from a rather simple challenge were enough to experience enjoyment.

\subsubsection{A Taxonomy of Game Graphics}

McLaughlin et al. [17] present a taxonomy of game graphics for serious games with a focus on learning based on graphical fidelity. They distinguish between different attributes of graphical content (Form, Motion, Materials \& Light) and include three levels of graphical fidelity (Simplified, Stylized, Naturalistic) that may influence how games affect players. Building on their approach and on our research results showing that the kind of graphic and their integration in the game has an impact on player experience, we suggest a categorization of graphics on a metalevel to further study the effects of graphical fidelity on player experience. Rather than using McLaughlin et al.'s [17] categorization of simplified, stylized, and naturalistic styles, we 
suggest a distinction based on the two game examples studied in this paper; low-fidelity (abstract) graphics, and high-fidelity (stylized) graphics. This taxonomy accounts for the fact that both function (embellishment, gameplay) and fidelity (abstract, stylized) of graphics need to be considered when assessing the impact of graphical fidelity on the player. It provides information on the level at which the interaction process of the game is affected (performance, usability, player experience). Results from the exploration of Rockband 2 show that players are not affected by visual fidelity if graphics only serve as embellishments. It is important to tie our findings back to work by Andersen et al. [1] suggesting that graphics that might be considered as embellishments (e.g., animations) do affect the player as soon as they are applied to convey feedback on the state of the game, thus turning them into graphics that are relevant to gameplay. For the dimension of gameplay, visual fidelity generally does not affect player performance unless stylized graphics add clutter to the game making it harder for the player to focus on relevant aspects.

An aspect that has not been studied yet is to what extent graphics that convey or reinforce premise affect player experience. Our results suggest that stylized graphics lead to increased relatedness and immersion, suggesting that the integration of dramatic elements of games through visuals may have a profound impact on the way games are perceived, which is in line with research results suggesting that dramatic elements of games influence the way players perceive themselves [3].

\subsection{Graphical Fidelity in Game Development}

In this section we demonstrate how our findings can be leveraged to improve the game development process. We provide examples of how a better understanding of the effects of visuals in games can be leveraged in the creation and distribution of games.

\subsubsection{Assigning Development Team Resources}

While it is important to note that this work in no way aims to diminish the contribution of graphic designers and the aesthetic value of visually pleasing game graphics, development resources are often limited and game development teams need to make informed decisions regarding the use of their resources [1]. Our results show that it can be feasible to include extremely simplistic, low-fidelity and low-cost graphics while still achieving a positive player experience, therefore allowing for a reduction of resources directed towards the graphical design of games. Additionally, user testing does not have to focus on in-game graphics at an early stage of the development process if we can predict these effects, and need not account for them at all stages of development. Finally, the results open the door for small independent studios without huge artistic resources, but with ideas for compelling mechanics to create successful casual games that push game design into the future.

\subsubsection{Marketing}

While simplistic graphics are generally accepted in projects aimed at casual gamers or mobile platforms, high-end graphics that push boundaries remain a selling point for AAA-projects targeted towards hardcore audiences, and many reviewing instances associated with the game development community (online communities, games magazines) heavily focus on graphical quality when assessing new games. In this context, graphics turn into a marketing tool with relevance beyond their impact on player experience: Although the experience while playing a game with well-designed mechanics may not be affected by graphical fidelity, the quality of graphics can be a crucial factor that affects which games players decide to buy. To further address this issue, future work should not only investigate effects of graphics on player experience, but also examine how different visuals may affect the overall appeal of games and the way that players rank their quality.

Understanding the relationship between graphics and player experience holds implications for the entire game development process. If effects of visuals on player experience can be predicted, the creation of prototypes featuring elaborate graphical designs as well as vertical slices for user testing can be replaced by functional prototypes allowing games user researchers to assess game mechanics and game play. Following this approach, the involvement of graphical artists in game development could shift. More development could happen in parallel, and artists could spend more of their time innovating in their craft, without concern for early integration into gameplay.

\subsubsection{Game Prototypes for Research}

Many researchers use game prototypes; some create games to study other topics in a low-risk manner (e.g., effects of distributed communication on collaboration [11]), whereas others use them to study topics in game research itself (e.g., the controlled analysis of player experience [3] or understanding game accessibility [12]). Our results suggest that if researchers choose simple mechanics for the games in their studies, they do not need to devote significant resources to the development of high-fidelity graphics.

\section{LIMITATIONS AND FUTURE WORK}

The games used in this study show some obvious limitations that indicate directions for future research. First, both games are 2D casual games with simple game mechanics - generalizing our findings to 3D games, as well as to different (and more complex) mechanics, are necessary. Second, we will extend our research to answer the question of how external graphics, which are not integrated with the game mechanic (i.e., game junk), influence the experience. Third, we are investigating the mediating effects of controller type (e.g., motion-based, realistic form factor) on the experience of game graphics. Finally, future work should address the impact of graphics on the dramatic elements of games; our findings suggest that abstract graphics that do not convey premise make it harder for the player to relate to them. This may be an interesting aspect as high-fidelity graphics could be applied to make the player develop a closer relationship with the game, e.g., with her avatar, or other in-game characters.

\section{CONCLUSION}

Broadening our understanding of how the visual design of games affects players is an important step towards arriving at a detailed understanding of player experience. In this paper, we demonstrate that the fidelity of graphics relevant for gameplay influences player experience on multiple levels. This broadens our understanding of how game graphics moderate other elements of player experience, and how they can be leveraged to influence the way players interact with games. Our findings hold implications for the casual game development community: First, we show that elaborate graphics are not always necessary to induce a positive player experience. Second, we demonstrate how different levels of visual fidelity can be applied to influence the player's experience with a game on a fundamental level. Third, we show how these findings can be integrated into the development process, and how they can be applied to help to inform the work of game designers wishing to create engaging experiences. 


\section{REFERENCES}

[1] Andersen, E., Lui, Y.-E., Snider, R., Szeto, R., and Popović, Z. Placing a Value on Aesthetics in Online Casual Games. In: Proceedings of CHI 2011, Vancouver, Canada (2011).

[2] Bateman, S., Mandryk, R. L., Gutwin, C., Genest, A., McDine, D., \& Brooks, C. (2010, April). Useful junk?: the effects of visual embellishment on comprehension and memorability of charts. In Proc. of the SIGCHI 2010.

[3] Birk, M. \& Mandryk, R.L. Control Your Game-Self: Effects of Controller Type on Enjoyment, Motivation, and Personality in Game. In Proc. of CHI 2013, France, (2013).

[4] Bracken, C.C., Botta, R.A.: Presence and Television: Form versus Content. In: Proceedings of the Fifth International Workshop on Presence, Porto, Portugal (2002).

[5] Bracken, C.C., Skalski, P.: Presence and video games: The impact of image quality and skill level. In: Proceedings of Presence 2006, Cleveland OH, USA (2006).

[6] Bracken, C.C., Skalski, P.: Telepresence and Video Games: The Impact of Image Quality. In: PsychNology Journal 7: 1 (2009), 101-112.

[7] Cheng, K., Cairns, P.A.: Behaviour, Realism and Immersion in Games. In: EA of CHI 2005, Portland, OR, USA (2005).

[8] Deternber, B.H., Simons, R.F., Reiss, J.E.: The Emotional Significance of Color in Television Presentations. In: Media Psychology 2: 4 (2000), 331-355.

[9] Ermi, L., Mäyrä, F.: Fundamental Components of the Gameplay Experience: Analysing Immersion. In: Proc. of DiGRA 2005, Vancouver, BC, Canada (2005).

[10] Fullerton, T., Game Design Workshop, 2nd Edition: A Playcentric Approach to Creating Innovative Games. Morgan Kaufmann, (2008).

[11] Gergle, D., Kraut, R.E., \& Fussell, S.R. (2006). The Impact of Delayed Visual Feedback on Collaborative Performance. In Proc. of CHI 2006, 1303-1312.

[12] Gerling, K.M., Livingston, I.J., Nacke, L.E., \& Mandryk, R.L. Full-Body Motion-based Game Interaction for Older Adults. In Proc. of CHI 2012, Austin, TX, USA (2012).

[13] Hart, S. G. NASA-Task Load Index (NASA-TLX); 20 Years Later. In Proc. of HFES (2006), 904-908.

[14] Hunicke, R., LeBlanc, M., \& Zubek, R. MDA: A formal approach to game design and game research. In Proc. of the AAAI Workshop on Challenges in Game AI 2004.
[15] Ivory, J.D., Kalyanaraman, S.: The Effects of Technological Advancement and Violent Content in Video Games on Players' Feelings of Presence, Involvement, Physiological Arousal, and Aggression. In: J Commun 57 (2007), 532-555.

[16] Krcmar, M., Farrar, K., McGloin, R.: The effects of video game realism on attention, retention, and aggressive outcomes. In: Comp Hum Beh 27 (2011), 432-439.

[17] McLaughlin, T., Smith, D., and Brown, I.A. A Framework for Evidence Based Visual Style Development for Serious Games. In: Proc. of FDG 2010, Monterey, USA (2010).

[18] Poels, K., de Kort, Y., IJsselsteijn, W. "It is always a lot of fun!" Exploring Dimensions of Digital Game Experience using Focus Group Methodology. In: Proc. of FuturePlay 2007, Toronto, Canada (2007).

[19] Przybylski, A.K., Weinstein, N., Murayama, K., Lynch, M.F., \& Ryan, R.M., The ideal-self at play: The appeal of video games that let you be all you can be. Psychological Science, 23 (1), (2011), 69-76.

[20] Ryan, R. M., Control and information in the intrapersonal sphere: An extension of cognitive evaluation theory. Journal of Personality and Social Psychology, 43, (1982), 450-461.

[21] Ryan, R.M., Rigby, C.S., Przybylski, A. The Motivational Pull of Video Games: A Self-Determination Theory Approach. In: Motivation and Emotion 30, 4 (2006).

[22] Ryan, R. M., \& Deci, E. L., Self-determination theory and the facilitation of intrinsic motivation, social development, and well-being. American Psychologist, 55, 2000b, 68-78.

[23] Smeddinck, J., Gerling, K.M., \& Tiemkeo, S. Visual Complexity, Player Experience, Performance and Physical Exertion in Motion-Based Games for Older Adults. In Proc. of ASSETS 2013, Bellevue, WA, USA.

[24] Sweetser, P., Wyeth, P.: GameFlow: A Model for Evaluating Player Enjoyment in Games. In: Computers in Entertainment 3, 3 (2005).

[25] Vidulich, M.A. and Tsang, P.S. Collecting NASA Workload Ratings: A Paper and Pencil Package. Working Paper. Moffett Field, CA: NASA Ames Research Center (1986).

[26] Watson, D., \& Clark, L. A. The PANAS-X: Manual for the positive and negative affect schedule-Expanded Form, (1994). 